\title{
The velocity field of ionized gas near Sgr A*
}

\author{
H. S. Liszt ${ }^{\star}$ \\ National Radio Astronomy Observatory, 520 Edgemont Road, Charlottesville, VA 22903-2475, USA \\ Received 28 February 2003 / Accepted 2 July 2003

\begin{abstract}
Several distinct kinematic patterns are superposed in the ionized gas seen nearest Sgr A*, the compact radio source at the center of the Milky Way. This has led to confusion over the morphology and motions of the gas; the recent dispersion-ring model of Sanders (1998) inadvertantly elides parts of two separate features and reproduces a description of the gas flow which does not actually occur. We demonstrate the gas kinematics via an analysis of the high-resolution H92 $\alpha$ radio recombination line data of Roberts \& Goss (1993) and Roberts et al. (1996). The characteristic kinematic pattern of the "Bar" is reproduced of ionized material in the Bar is $\sim 27 M_{\odot}$. The Bar and Eastern Arm together probably represent a single stream of gas falling on to the galactic center, crossing from east to west.
\end{abstract} \\ by rotation in near-polar orbits seen edge-on at distances of $0.3-0.8 \mathrm{pc}$ from Sgr $\mathrm{A}^{*}$, with larger radii seen to the east. The mass
}

Key words. Galaxy: center - radio lines: ISM

\section{Introduction}

When the kinematics of the gas in the inner few pc of the galaxy first became accessible, they were studied with an eye to derivation of the mass distribution and demonstration of the existence of a massive black hole (Lo \& Claussen 1983; Brown \& Liszt 1984; Genzel \& Townes 1987; Sanders 1992), presumably the compact source Sgr A* discovered by Balick \& Brown (1974). Now, that role seems better occupied by study of proper motions of the bright stars in the central stellar cluster (Ghez et al. 1998, 2000; Eckart et al. 2002; Schödel et al. 2002; Ghez et al. 2003). But the gas kinematics remain of interest because of the large reservoir of material near the central black hole and the possibility that it will feed star formation and other nuclear activity (Morris \& Serabyn 1996).

Although the gas kinematics are relatively well understood in the small - line profiles are available with relatively good spatial and/or velocity resolution - a consensus description of gas flow near $\mathrm{Sgr} \mathrm{A}^{*}$ has been somewhat slower to emerge; compare the models of Quinn \& Sussman (1985), Lacy et al. (1991) and Sanders (1998). The dispersion-ring models of Sanders (1998) are especially attractive, in demonstrating that the observed gas flows need not be short-lived (Lo \& Claussen 1983). Unfortunately, his model of the ionized gas reproduces a description of the velocity field (ibid) which was superseded by more recent observational material (Lacy et al. 1991; Roberts \& Goss 1993; Roberts et al. 1996). The model elides two features which are actually distinct (the Bar and Northern Arm;

\footnotetext{
* e-mail: hliszt@nrao.edu
}

see Sect. 3) and does not incorporate the kinematics which are actually observed in either.

The purpose of the present work is to show the motions of the gas clearly enough that this sort of confusion does not persist, and to aid in formulation of a more definitive model. However, we also provide an elementary interpretation of the gas geometry and kinematics; rotation in close-in $(0.3-0.8 \mathrm{pc})$ near-polar orbits, in the gravitational potential imposed by the known mass distribution, very simply reproduces much of the behaviour. Section 2 describes the observational material, Sect. 3 discusses the gas motions, and an elementary interpretation is provided in Sect. 4 .

\section{Observational material}

The motions of the gas near Sgr $\mathrm{A}^{*}$ are most clearly distinguished in the $\lambda 12.8 \mu$ [Ne II] line, most recently and comprehensively studied by Lacy et al. (1991), and in radio recombination line work like that analyzed here, the H92 $\alpha$ mapping of Roberts \& Goss (1993) and Roberts et al. (1996) ${ }^{1}$ (see also Schwarz et al. 1989). Lower velocity-resolution observations of the gas near $\mathrm{Sgr} \mathrm{A}^{*}$ have also been performed in the Br $\gamma$ line by Herbst et al. (1993), which was cited by Sanders (1998).

Some explanation of these datasets is in order. The [Ne II] observations (at $2^{\prime \prime}$ resolution) show the motions of all the ionized gas, but at somewhat low velocity resolution

\footnotetext{
1 These datasets are publicly available at imagelib.ncsa.uiuc.edu/project/document/95.DR.01 and imagelib.ncsa.uiuc.edu/project/document/95.DR.03
} 


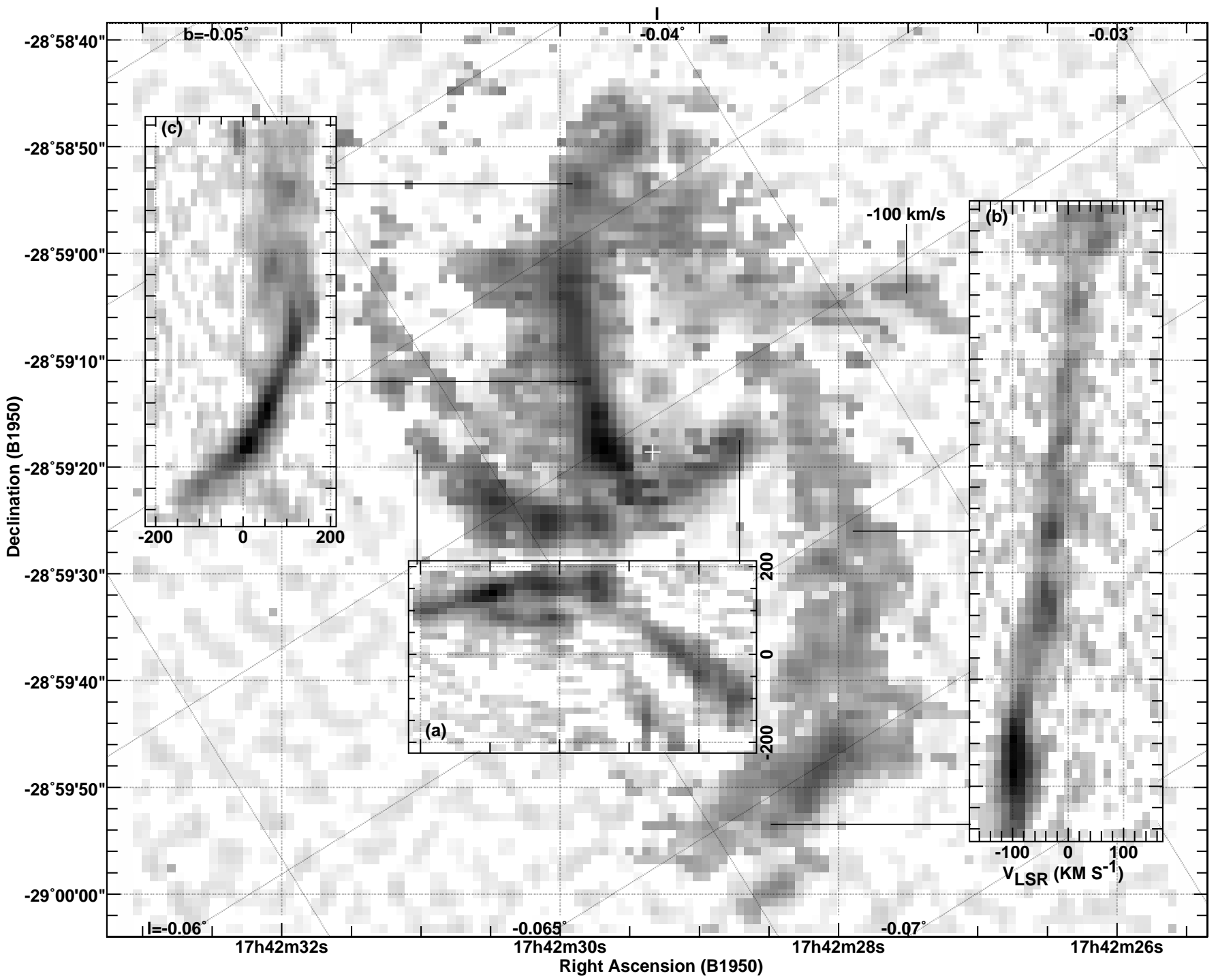

Fig. 1. A map of the integrated H92 $\alpha$ emission at 2" resolution (Roberts \& Goss 1993), and superposed position-velocity diagrams along the ridge lines of the major morphological features. The position-velocity diagrams represent: a) the "Bar" and (at the extreme east) "Eastern Arm"; b) the Western Arm, which is the ionized inner edge of the molecular circumnuclear disk; c) the Northern Arm. This display covers only the range $-200 \leq v \leq+200 \mathrm{~km} \mathrm{~s}^{-1}$ and misses some gas near $\mathrm{Sgr} \mathrm{A}^{*}$ which is recovered in Fig. 2.

$\left(30 \mathrm{~km} \mathrm{~s}^{-1}\right)$ compared to the radio data $\left(14 \mathrm{~km} \mathrm{~s}^{-1}\right)$. The earlier $\mathrm{H} 92 \alpha$ radio dataset (at $2^{\prime \prime}$ resolution) was limited to $-200 \leq$ $v \leq+200 \mathrm{~km} \mathrm{~s}^{-1}$ and the full extent of the negative velocity gas was recovered (at $1^{\prime \prime}$ resolution) only by Roberts et al. (1996). The Br $\gamma$ observations have very low velocity resolution $\left(100 \mathrm{~km} \mathrm{~s}^{-1}\right)$ and show that $\mathrm{Br} \gamma$ emission arises in only a limited part of the gas.

Figure 1 is an attempt to display simulaneously the disposition and motion of the ionized gas. What has been done is to superpose position-velocity diagrams, formed along the ridge lines of the major features, on a spatial map of the integrated intensity over the range $-200 \leq v \leq+200 \mathrm{~km} \mathrm{~s}^{-1}$. The ridge lines are smoothly curving arcs and the position-velocity diagrams are aligned with the regions sampled so that the kinematics and spatial appearance can be associated.

Figure 2 shows the kinematics of the negative-velocity gas lacking in Fig. 1, in the southern extension of the Northern
Arm. Comparison of the panel at lower right in Fig. 2. with the Br $\gamma$ data (Figs. 2 and 3 of Herbst et al. 1993) shows that the $\mathrm{Br} \gamma$ line arises exclusively in the Northern Arm, not in the Bar.

\section{Motions of the ionized gas near Sgr $\mathbf{A}^{*}$}

Naming conventions descend to us from early work showing the detailed structure of the ionized gas in Sgr A (west) (Ekers et al. 1983; Lo \& Claussen 1983), which is said to reside in a pattern called the "mini-spiral". Figure 1 of Lacy et al. (1991) explicitly labels the various constituents of the gas and the caption to Fig. 1 here reiterates the nomenclature.

\subsection{Kinematic patterns in the Bar, Northern Arm and Eastern Arm}

Position-velocity diagram a in Fig. 1 traces the ionized gas from the western terminus of the so-called Bar, just inside 


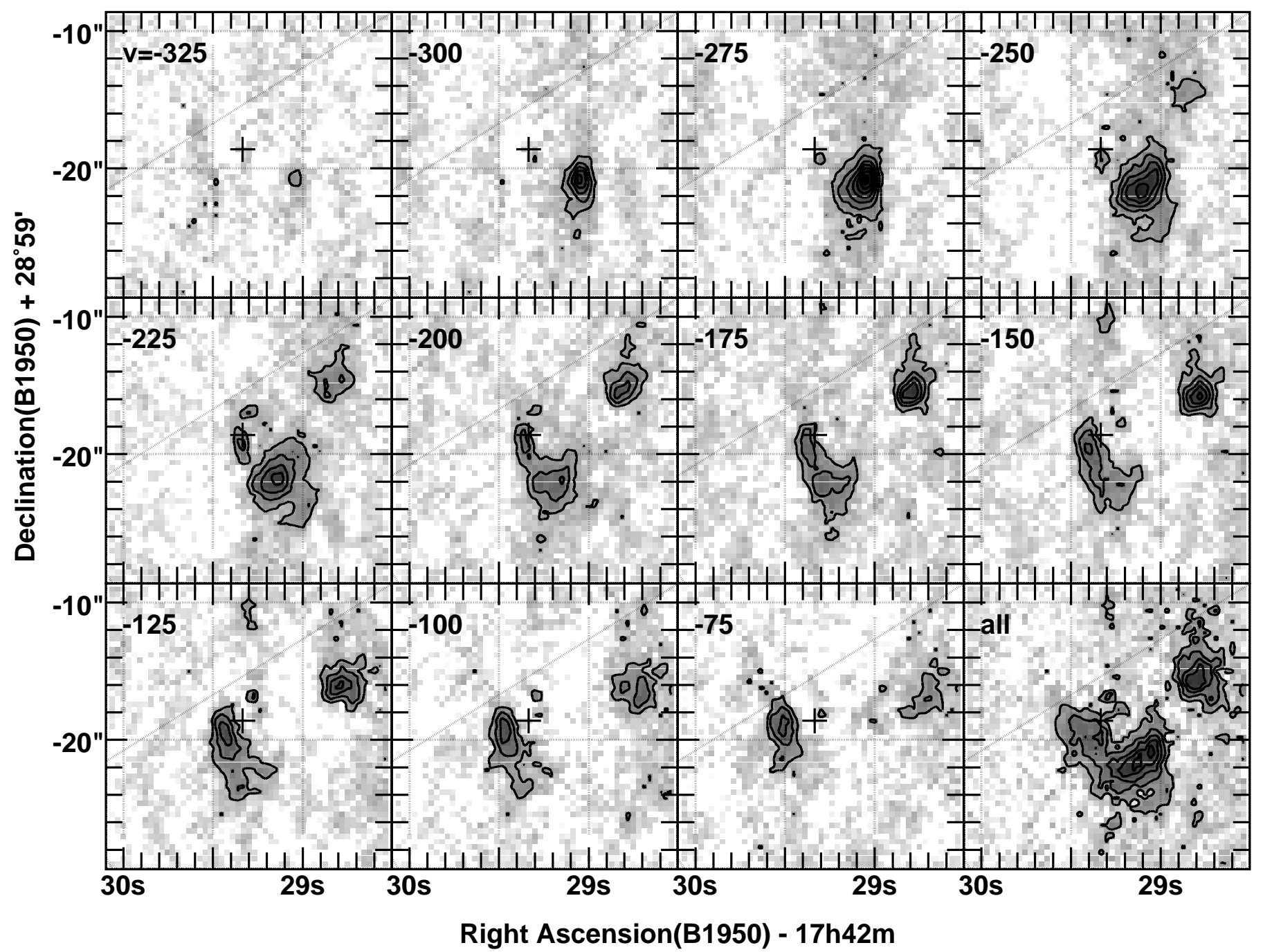

Fig. 2. H92 $\alpha$ emission (Roberts et al. 1996) integrated over $25 \mathrm{~km} \mathrm{~s}^{-1}$-wide velocity intervals centered as indicated with contours at 0.02 , 0.04 , $0.06 \ldots \mathrm{Jy} \mathrm{beam}^{-1} \mathrm{~km} \mathrm{~s}^{-1}$ and (lower right) over all velocities with contours at $0.04,0.08,0.12 \ldots \mathrm{Jy} \mathrm{beam}^{-1} \mathrm{~km} \mathrm{~s}^{-1}$. The spatial resolution is $1^{\prime \prime}$.

the inner edge of the circumnuclear disk (CND), into the Eastern Arm. Superposition of unrelated gas features causes two kinematic components to appear at most positions along the ridge line. The pattern associated with the Bar is that seen at higher velocity at each position. There is a smooth change from $-100 \mathrm{~km} \mathrm{~s}^{-1}$ at far west, where the line is rather broad, as expected for a ring seen tangent to the line of sight; through zero velocity a few arcseconds west of $\mathrm{Sgr} \mathrm{A}^{*}$ at what is probably the point of closest approach to the center (i.e. draw the normal to the ridge line which passes through Sgr $\mathrm{A}^{*}$ ); to $150 \mathrm{~km} \mathrm{~s}^{-1}$ just east of $\mathrm{Sgr} \mathrm{A}^{*}$, and then to gradually lower velocity where the ridge line curves up into the Eastern Arm. This is the inherent velocity pattern of the Bar and Eastern Arm, with velocity increasing monotonically from west to east across the position of $\mathrm{Sgr} \mathrm{A}^{*}$. The negative-velocity gas just south and west of Sgr A* in panel a arises in the southern extension of the Northern Arm, not in the Bar, as shown in Fig. 2.

Position-velocity diagram $\mathrm{c}$ in Fig. 1 traces the prominent Northern Arm. The line profile narrows and brightens and the velocity changes rapidly across the declination of Sgr A*, exiting the passband of the dataset to the south where it crosses the
Bar. The behaviour of this gas is followed in Fig. 2, drawn from the $1^{\prime \prime}$ resolution, negative-velocity $\mathrm{H} 92 \alpha$ dataset of Roberts et al. $(1996)^{2}$.

In Fig. 2 there are two separate sets of behaviour, one of which represents the southern terminus of the Northern Arm. Close to Sgr A* the Northern Arm loops around to form the "Mini-Cavity" (ibid) which is the space inside the crook of the integrated flux distribution just south and west of Sgr A*. Velocities of (about) $-125 \mathrm{~km} \mathrm{~s}^{-1}$ are seen to the east of Sgr A*, $-200 \mathrm{~km} \mathrm{~s}^{-1}$ below it, and $-275 \mathrm{~km} \mathrm{~s}^{-1}$ to the immediate west. This is the negative-velocity gas just south of Sgr A* seen in panel a of Fig. 1. The pattern of the negative velocity gas close to Sgr A* is identical to that of the $\mathrm{Br} \gamma$ emission (Herbst et al. 1993), implying that it arises in the Northern Arm.

The gas which peaks at $-150 \mathrm{~km} \mathrm{~s}^{-1}$ near the western edge of Fig. 2 represents the unrelated western edge of the Bar. Emission from the Bar in Fig. 2 moves little with velocity; perhaps $2^{\prime \prime}$ to the south over almost $200 \mathrm{~km} \mathrm{~s}^{-1}$. This is a reflection

\footnotetext{
2 We removed linear spectral baselines from this datacube which greatly improved the appearance of the channel maps.
} 


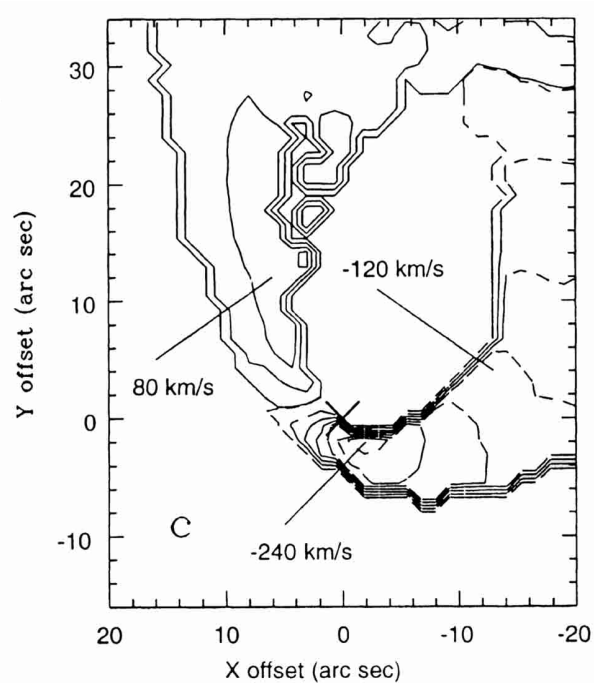

Fig. 3. From Sanders (1998), Fig. 7 panel c), the model velocity field for the Northern Arm and Bar. Used by permission.

of the broad profile seen at the very end of the Bar in panel a of Fig. 1, but Fig. 2. shows that the profile is in fact even a bit broader yet.

\subsection{Some marginal details about the Bar}

The Bar has something of an intensity minimum about where it crosses the Northern Arm, which is perhaps more apparent in the position-velocity diagram than in the integrated intensity. Also, the emission profile is double to the east, but the lowervelocity component does not show the velocity gradient which is characteristic of the Bar. Much of the emission in this region occurs at $+100 \mathrm{~km} \mathrm{~s}^{-1}$ and the line-doubling could result from superposition of another kinematic component.

There is unexplained and anomalous emission at $-100 \mathrm{~km} \mathrm{~s}^{-1}$ at the location so remarked in Fig. 1; the presence of negative-velocity emission in this region was noted by Lo \& Claussen (1983). It lies along an extrapolation of the figure of the western end of the Bar and seems nominally consistent with an origin in the same model geometry which reproduces the Bar gas.

\subsection{Gas flow in the model of Sanders (1998)}

Figure 3 reproduces panel c of Fig. 7 in Sanders (1998) showing the gas flow in his dispersion-ring model of the so-called "Extended Northern Arm". The velocity field in Sanders' work conforms to the schematic description of Fig. 3 in Lo \& Claussen (1983), but there is no component of the gas which actually behaves in this way to the west of $\mathrm{Sgr} \mathrm{A}^{*}$. The $-240 \mathrm{~km} \mathrm{~s}^{-1}$ gas seen below Sgr A* is indeed the bottom of the Northern Arm, but (as shown in Fig. 2) its velocity becomes more negative to the west, forming the small "mini-cavity". Conversely, the gas which appears at $-120 \mathrm{~km} \mathrm{~s}^{-1}$ at the western terminus of the Bar is at high positive velocity near Sgr A* (Fig. 1, panel a). Sanders' model orphans both the eastern half

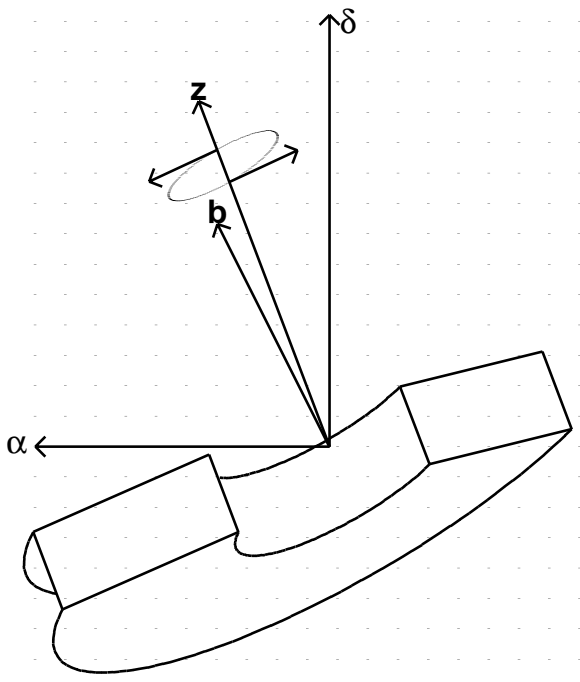

Fig. 4. View of the model ring segment described in Sect. 4 of the text. The inner and outer radii are 0.3 and $0.8 \mathrm{pc}$. The model is inclined $76^{\circ}$ to the line of sight in position angle $25^{\circ}$ and extends $\pm 0.09 \mathrm{pc}$ about its midplane. Gas in the model rotates about the normal to the disk, labelled "z", flowing from east to west behind Sgr A*. The projection of the local normal to the galactic equator - the axis of normal galactic rotation - is labeled " $b$ ".

of the Bar and the most negative-velocity gas in the southern extension of the Northern Arm.

\section{A simple geometrical model for the Bar gas}

A variety of models have been proposed for various portions of the Northern Arm but there has as yet been no model proposed for the behaviour of the Bar described in Sect. 3. A unique dynamical description of any gas motion may be impossible. Here we provide a kinematic description akin to that which first defined the properties of the CND (Brown \& Liszt 1984; Liszt et al. 1985; Güsten et al. 1987).

We consider circular motion in equilibrium with the gravitational potential resulting from the presence of a central black hole, of mass $2.65 \times 10^{6} M_{\odot}$, and the central stellar cluster. For the (spherical) cluster model we adopt a density law $\rho_{*}(R) \propto R^{-1.7}$ (Allen et al. 1983) and normalize the total mass so as to give a circular velocity of $110 \mathrm{~km} \mathrm{~s}^{-1}$ at $R=2 \mathrm{pc}$ as demanded by the CND kinematics. The Sun-center distance is taken as $8.5 \mathrm{kpc}$.

We adopt a planar ring geometry with a line of sight inclination of $76^{\circ}$, and assign a position angle $25^{\circ}$ in the usual sense rotated counter-clockwise from celestial north (this orientation is nearly polar). We consider a disk of uniform density and temperature within $\pm 0.09 \mathrm{pc}$ of its midplane $\left(0.09 \mathrm{pc}=2.2^{\prime \prime}\right)$ over a $150^{\circ}$ arc on the far side of the galactic center, mostly to the East. There is no obvious front-back symmetry to the Bar nor is it symmetrically disposed east-west about Sgr $\mathrm{A}^{*}$. The frontback placement relative to $\operatorname{Sgr} \mathrm{A}^{*}$ (the sense of the tilt of the inclination out of the plane of the sky) is a priori ambiguous. Our assignment of the far distance (back side) is made because, with this orientation, the gas flows in the same sense that it nears the center (see just below). This model geometry, shown 


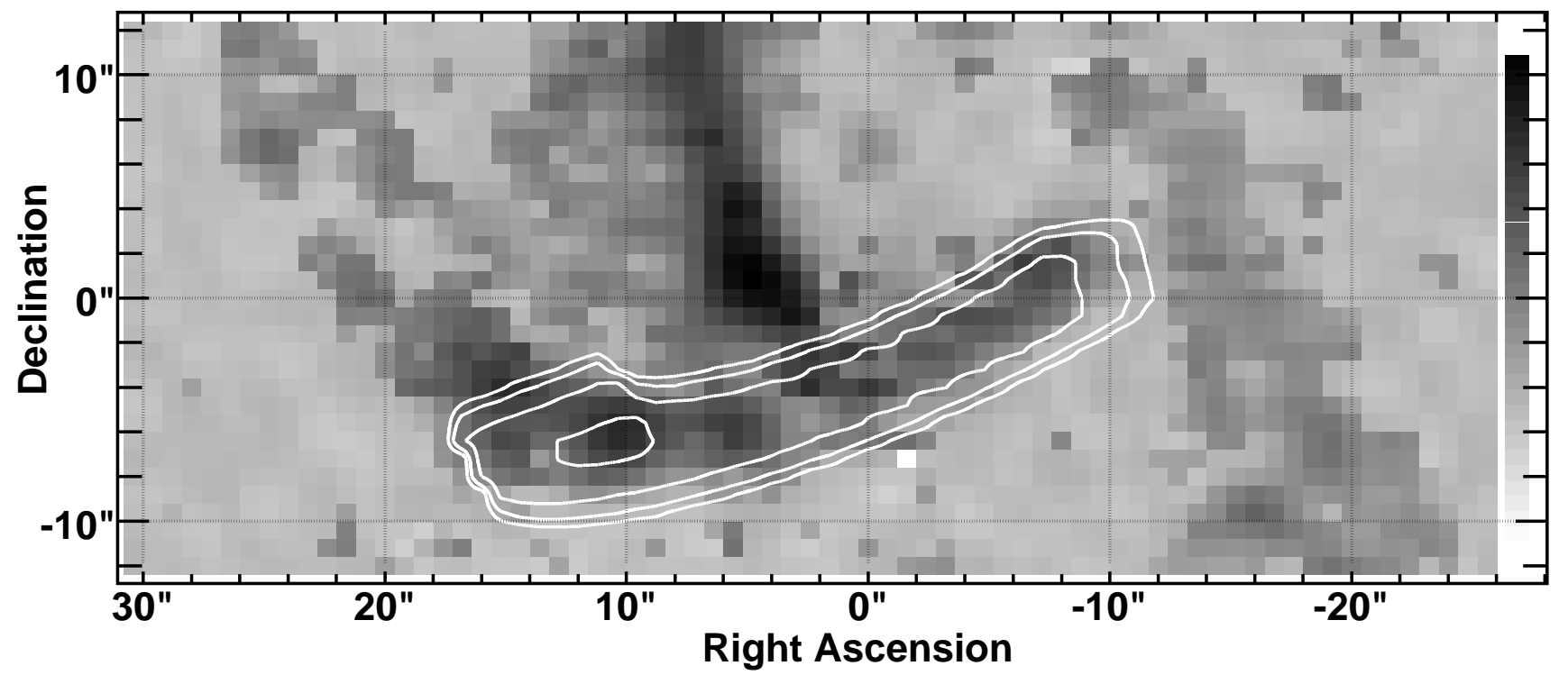

Fig. 5. Simulated H92 $\alpha$ emission from a flat ring segment extending $0.35-0.75 \mathrm{pc}$ from the galactic center in a $150^{\circ}$ arc, shown in contours projected against a grayscale representation of the emission from the Bar and Eastern Arm. Coordinates are relative to Sgr A*.

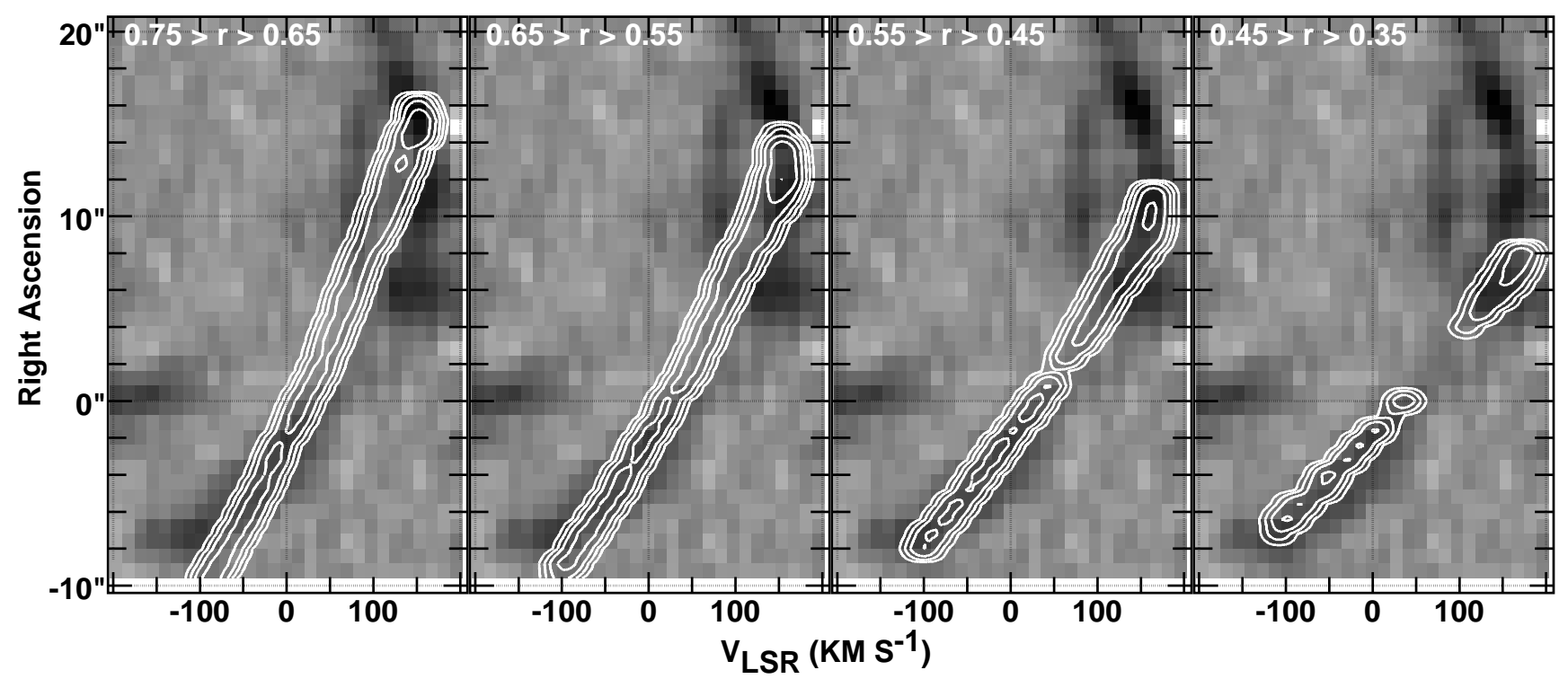

Fig. 6. Position-velocity diagrams for a series of nested, coplanar, thin rings of gas in circular motion, shown as white contours projected against H92 $\alpha$ emission traced along the Bar and Eastern Arm as in Fig. 1a. Right ascension is relative to Sgr A*. In each panel, the radial extent of the ring (in pc) is indicated at the upper left. All the rings lie in a single plane which is inclined $76^{\circ}$ to the line of sight and has its major axis at position angle $25^{\circ}$ measured counterclockwise from celestial north, as shown in Fig. 4.

in Fig. 4, should be thought of merely as an enclosing outline. The radial decomposition in Fig. 6 is a guide as to which parts of it are really populated.

We modelled emission from the disk by integrating the equation of radiative transfer (Gordon 1988; Roelfsema \& Goss 1992) along the line of sight in three dimensions at $0.5^{\prime \prime}$ intervals and convolved these profiles over $2^{\prime \prime}$ Gaussian beams (as observed) spaced on an $0.8^{\prime \prime}$ grid (also as observed). The intrinsic velocity dispersion of the gas was taken as $12 \mathrm{~km} \mathrm{~s}^{-1}$ and the profiles were calculated in channels of separation $6 \mathrm{~km} \mathrm{~s}^{-1}$. The gas is optically thin and the model is uniform, so path length effects and geometry together determine the shapes of the line profiles.
The integrated emission from a fully-filled ring model is shown projected against the data in Fig. 5. Position-velocity diagrams along the ridge line of the Bar are shown in Fig. 6 for individual, non-overlapping radial intervals. The model emission is superposed as white contours on the data which is shown as a grayscale as in Fig. 1a. The gray-scale background in Fig. 6 is the same in each panel and follows the same track as in panel a of Fig. 1 but the zero-level has been made somewhat darker to highlight the contours.

Figure 6 shows the kinematics decomposed radially; bear in mind that the motion is pure rotation about a single axis and spatial structure arises solely from the projected geometry. Comparison of the various panels suggests that gas at larger 
radii exists primarily to the east. To the west, no gas is really required at $R>0.45 \mathrm{pc}$ and the central gap indicates that little is in fact present. In this view, gas is spiraling in from the east, crossing the center from east to west, and it is on this basis that we preferred to assign the far distance to the Bar; the kinematic modelling is unaffected by this distinction because all motion is circular. Note that our partial disk model does not produce an extremely broad line profile at the western edge of the Bar, which is an important distinguishing characteristic. This is an artifact of the simplicity of the adopted geometry, in which ring segments of constant azimuthal extent are used at all radii.

Flow in the Northern Arm also loops around the center from back to front (Yusef-Zadeh et al. 1998a,b) but much more compactly. Note that the eastern side of the CND is generally considered to be the more distant one (Liszt et al. 1985), so material dropping out of it would approach the center from behind, moving toward the Sun.

It seems clear that one could follow the emission further into the Eastern Arm using a prescription of this sort, but not so obviously in the same plane. The implication is that a parcel of gas is dropping onto the center in progressively more nearly polar orbits after a drastic change in angular momentum, but with details still to be specified. To reproduce this behaviour, the Bar and Eastern Arm should be viewed as a single structure.

\subsection{Mass of ionized gas in the Bar}

Gordon (1988) and Roelfsema \& Goss (1992) give expressions for the source function of the H92 $\alpha$ transition, which varies as $n_{\mathrm{e}}^{2} / T_{\mathrm{e}}{ }^{2.5}$. The mass of the Bar is found by integrating the model brightness temperature profiles, matching the total to that which is observed, and then integrating the electron density so derived over the model volume. In this way, we find $M_{\mathrm{Bar}}=27\left(T_{\mathrm{e}} / 7000 \mathrm{~K}\right)^{3 / 2}\left(10000 \mathrm{~cm}^{-3} / n_{\mathrm{e}}\right) M_{\odot}$, where the parametrized electron temperature and density are typical values. The total emission integrated over the entirety of Fig. 1 is 6.3 times greater than that in the Bar alone, and the total inferred mass of ionized gas, some $160 M_{\odot}$, is about $1 \%$ of the mass seen in the neutral/molecular regions of the larger CND.

\section{Summary}

We displayed the kinematics of ionized gas along the various features seen near Sgr A*, in order to distinguish the kinematics of one more or less connected ridge of gas which is commonly known as the Bar and Eastern Arm. The gas motions seen near Sgr A* are complicated by the coincidental superposition of several features and the kinematics of the Bar have been obscure for much of the last $20 \mathrm{yrs}$. An extant dynamical model of its gas flow reproduces an obsolete description of the kinematics and must be recast.
We showed how the motion of the Bar gas can be reproduced by rotation at the local circular velocity within a series of thin coplanar, near-polar rings seen almost edge-on at radii $R=0.3-0.8 \mathrm{pc}$. Detailed comparison of the emission distribution and a radial decomposition of these motions suggests that the Bar gas is spiralling in across the center from east to west, just on the far side of the galactic center. The mass of ionized gas in the Bar is $M_{\mathrm{bar}} \sim 27 M_{\odot}$.

Acknowledgements. The NRAO is operated by AUI, Inc. under a cooperative agreement with the US National Science Foundation. I thank D. Roberts for making the radio recombination line datasets available, B. Sanders for his comments and long friendship, and the referee, again D. Roberts, for his constructive and informative remarks.

\section{References}

Allen, D. A., Hyland, A. R., \& Jones, T. J. 1983, MNRAS, 204, 1145 Balick, B., \& Brown, R. L. 1974, ApJ, 194, 265

Brown, R. L., \& Liszt, H. S. 1984, ARA\&A, 22, 223

Eckart, A., Genzel, R., Ott, T., \& Schödel, R. 2002, MNRAS, 331, 917

Ekers, R. D., van Gorkom, J. H., Schwarz, U. J., \& Goss, W. M. 1983, A\&A, 122, 143

Genzel, R., \& Townes, C. H. 1987, ARA\&A, 25, 377

Ghez, A. M., Duchêne, G., Matthews, K., et al. 2003, ApJ, 586, L127

Ghez, A. M., Klein, B. L., Morris, M., \& Becklin, E. E. 1998, ApJ, 509,678

Ghez, A. M., Morris, M., Becklin, E. E., Tanner, A., \& Kremenek, T. 2000, Nature, 407, 349

Gordon, M. A. 1988, in Galactic and Extragalactic Radio Astronomy, ed. G. L. Verschuur, \& K. I. Kellermann (New York: Springer), 37

Güsten, R., Genzel, R., Wright, M. C. H., et al. 1987, ApJ, 318, 124

Herbst, T. M., Beckwith, S. V. W., Forrest, W. J., \& Pipher, J. L. 1993, AJ, 105, 956

Lacy, J. H., Achtermann, J. M., \& Serabyn, E. 1991, ApJ, 380, L71

Liszt, H. S., Burton, W. B., \& van der Hulst, J. M. 1985, A\&A, 142, 237

Lo, K. Y., \& Claussen, M. J. 1983, Nature, 306, 647

Morris, M., \& Serabyn, E. 1996, ARA\&A, 34, 645

Quinn, P. J., \& Sussman, G. J. 1985, ApJ, 288, 377

Roberts, D. A., \& Goss, W. M. 1993, ApJS, 86, 133

Roberts, D. A., Yusef-Zadeh, F., \& Goss, W. M. 1996, ApJ, 459, 627

Roelfsema, P. R., \& Goss, W. M. 1992, A\&ARv, 4, 161

Sanders, R. H. 1992, Nature, 359, 131

Sanders, R. H. 1998, MNRAS, 294, 35

Schödel, R., Ott, T., Genzel, R., et al. 2002, Nature, 419, 694

Schwarz, U. J., Bregman, J. D., \& van Gorkom, J. H. 1989, A\&A, 215, 33

Yusef-Zadeh, F., Roberts, D. A., \& Biretta, J. 1998a, ApJ, 499, L159

Yusef-Zadeh, F., Roberts, D. A., \& Biretta, J. 1998b, ApJ, 503, L191 